\title{
Barrier, Adsorptive, and Mechanical Properties of Containers Molded from PET/PP Blends for Use in Pharmaceutical Solutions
}

\author{
Tadashi Otsuka ${ }^{1}$, Ayako Kurosawa ${ }^{1}$, Masaki Maeda ${ }^{1}$, Kazushi Yamada ${ }^{2}$, Masuo Murakami², \\ Hiroyuki Hamada ${ }^{2}$ \\ ${ }^{1}$ Pharmaceutical Development Research Laboratories of Senju Pharmaceutical Co., Ltd., Kobe, Japan; ${ }^{2}$ Department of Advanced \\ Fibro-Science, Kyoto Institute of Technology, Kyoto, Japan. \\ Email: tadashi-ootsuka@senju.co.jp
}

Received August $5^{\text {th }}$, 2013; revised September $9^{\text {th }}$, 2013; accepted September 21 $1^{\text {st }}, 2013$

Copyright (C) 2013 Tadashi Otsuka et al. This is an open access article distributed under the Creative Commons Attribution License, which permits unrestricted use, distribution, and reproduction in any medium, provided the original work is properly cited.

\begin{abstract}
In this study, the adsorptive properties of L-menthol, moisture vapor transmission rate, and mechanical properties of poly (ethylene terephthalate) (PET), polypropylene (PP), and their blends were evaluated in containers fabricated by injection molding without a compatibilizing agent. These containers intended to hold pharmaceutical solutions containing lipophilic chemicals such as L-menthol. By the addition of a small amount of PP, the moisture barrier properties of PET were effectively improved, but its anti-adsorptive property was reduced. The strength of the PET/PP blends was reduced, but some PET/PP blends were still able to bear the experimental load required for application in eye-drop containers. $\mathrm{PET} / \mathrm{PP}=9 / 1$ and $\mathrm{PET} / \mathrm{PP}=8 / 2$ were found to be most applicable for using in the fabrication of containers for medicinal solutions.
\end{abstract}

Keywords: PET/PP; Blend; Barrier; Adsorptive; Mechanical Properties

\section{Introduction}

In Japan, L-menthol is preferably included as an algefacient in over-the-counter eye drops [1]. Because L-menthol is a hydrophobic compound, it has a tendency to be adsorbed onto the walls of eye-drop containers, which are commonly made of polyolefin resin. In contrast, hydrophobic compounds are hardly adsorbed onto hydrophilic polyester resins such as poly (ethylene terephthalate) (PET) [2]. Therefore, PET is suitable for eye-drop containers including L-menthol. However, it is well known that PET has a high moisture vapor transmission rate (MVTR) [3], i.e., a low vapor barrier property, which results in a short shelf-life of the product [4]. Because polypropylene (PP) exhibits a low MVTR [5], a blend of PET and PP might compensate for the disadvantage of each material and afford an innovative material for eye-drop containers.

The mechanical and thermal properties of PET/PP blends have been widely reported [6]. Nevertheless, there is very little information in literature regarding the adsorptive and barrier properties of PET/PP blends. Fur- thermore, studies have reported the improvement of mechanical characteristics of PET/PP blends by the addition of compatibilizing agents [7-11]. However, there are safety issues associated with the use of these agents in containers for pharmaceuticals. Therefore, in this study, the adsorptive, barrier, and mechanical properties of PET/PP blends in eye-drop containers fabricated without the addition of a compatibilizing agent were investigated.

\section{Experimental}

\subsection{Materials}

Isophthalic PET resin "MA1340P" with an intrinsic viscosity of $0.57 \mathrm{dL} / \mathrm{g}$ was supplied by Unitika Ltd., Japan. Random copolymer PP resin "MG03E" with a melt flow rate of $30 \mathrm{~g} / 10$ min was supplied by Japan Polypropylene Corporation, Japan. The properties of PET and PP resins are listed in Tables $\mathbf{1}$ and 2, respectively.

\subsection{Sample Preparation}

Table 3 lists the composition of PET/PP blends used in 
Table 1. Properties of the PET resin used.

\begin{tabular}{cc}
\hline Properties & Value \\
\hline Intrinsic viscosity (IV) & $0.57 \mathrm{dL} / \mathrm{g}$ \\
Glass-transition temperature & $72^{\circ} \mathrm{C}$ \\
Melting point & $231^{\circ} \mathrm{C}$ \\
Density & $1.38 \mathrm{~g} / \mathrm{cm}^{3}$ \\
Tensile modulus & $1.2 \mathrm{GPa}$ \\
Flexural modulus & $2.3 \mathrm{GPa}$ \\
Flexural strength & $67 \mathrm{MPa}$ \\
Izod impact strength & $26.0 \mathrm{~J} / \mathrm{m}$ \\
Rockwell hardness (R scale) & 111 \\
Heat distortion temperature & $65^{\circ} \mathrm{C}$ \\
\hline
\end{tabular}

Table 2. Properties of the PP resin used.

\begin{tabular}{cc}
\hline Properties & Value \\
\hline Melt flow rate $\left(230^{\circ} \mathrm{C}\right)$ & $30 \mathrm{~g} / 10 \mathrm{~min}$ \\
Spiral flow test & $850 \mathrm{~mm}$ \\
Density & $0.90 \mathrm{~g} / \mathrm{cm}^{3}$ \\
Tensile modulus & $1.2 \mathrm{GPa}$ \\
Tensile strength & $27 \mathrm{MPa}$ \\
Flexural modulus & $1.15 \mathrm{GPa}$ \\
Flexural strength & $33 \mathrm{MPa}$ \\
Charpy impact strength & $4.0 \mathrm{~kJ} / \mathrm{m}^{2}$ \\
Rockwell hardness (R scale) & 90 \\
Heat distortion temperature & $85^{\circ} \mathrm{C}$ \\
\hline
\end{tabular}

Table 3. Compositions of the PET/PP blends.

\begin{tabular}{ccc}
\hline Sample & PET & PP \\
\hline PET & $100 \mathrm{wt} \%$ & $0 \mathrm{wt} \%$ \\
PET/PP $=9 / 1$ & $90 \mathrm{wt} \%$ & $10 \mathrm{wt} \%$ \\
PET/PP = 8/2 & $80 \mathrm{wt} \%$ & $20 \mathrm{wt} \%$ \\
PET/PP = 7/3 & $70 \mathrm{wt} \%$ & $30 \mathrm{wt} \%$ \\
PET/PP = 5/5 & $50 \mathrm{wt} \%$ & $50 \mathrm{wt} \%$ \\
PET/PP = 3/7 & $30 \mathrm{wt} \%$ & $70 \mathrm{wt} \%$ \\
PP & $0 \mathrm{wt} \%$ & $100 \mathrm{wt} \%$ \\
\hline
\end{tabular}

this study. PET and PP were dried using a dehumidifying drier at $110^{\circ} \mathrm{C}$ for $16 \mathrm{~h}$ and at $80^{\circ} \mathrm{C}$ for $16 \mathrm{~h}$, respectively. The raw materials were then compounded by a twin screw extruder (LABOTEX30HSS, Japan Steel Works
Ltd., Japan); the compounding conditions are given in Table 4. The compounded materials were then pelletized by a pelletizing machine. The pellets were dried in a convection oven set at $90^{\circ} \mathrm{C}$ for $16 \mathrm{~h}$ before injection molding to form cylindrical bottle specimens, as shown in Figure 1. Dumbbell-shaped specimens [Type A of JIS K7139 (ISO3167: 1993)] were formed using a PoYuen UM50 injection molding machine. The nozzle specimens for the eye-drop container were formed using a FANUK ROBOSHOT a50 injection molding machine, as shown in Figure 2.

\section{Characterization}

\subsection{MVTR Measurement}

Each bottle specimen was filled with $5 \mathrm{~mL}$ purified water

Table 4. Compounding conditions.

\begin{tabular}{ccc}
\hline Sample & Barrel temperature & Screw speed \\
\hline $\mathrm{PET} / \mathrm{PP}=9 / 1$ & $230^{\circ} \mathrm{C}-245^{\circ} \mathrm{C}$ & $250 \mathrm{rpm}$ \\
$\mathrm{PET} / \mathrm{PP}=8 / 2$ & $230^{\circ} \mathrm{C}-245^{\circ} \mathrm{C}$ & $250 \mathrm{rpm}$ \\
$\mathrm{PET} / \mathrm{PP}=7 / 3$ & $230^{\circ} \mathrm{C}-245^{\circ} \mathrm{C}$ & $250 \mathrm{rpm}$ \\
$\mathrm{PET} / \mathrm{PP}=5 / 5$ & $210^{\circ} \mathrm{C}-240^{\circ} \mathrm{C}$ & $265 \mathrm{rpm}$ \\
$\mathrm{PET} / \mathrm{PP}=3 / 7$ & $210^{\circ} \mathrm{C}-240^{\circ} \mathrm{C}$ & $275 \mathrm{rpm}$ \\
\hline
\end{tabular}

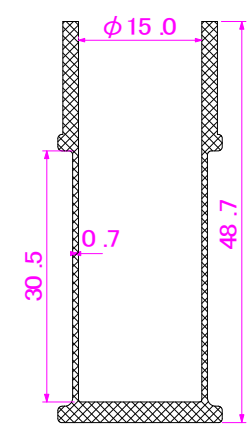

Figure 1. Cylindrical bottle specimen.

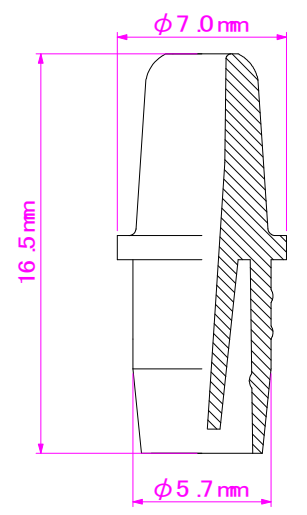

Figure 2. Nozzle specimen. 
and then closed with a low-density polyethylene (LDPE) sealing cap, as illustrated in Figure 3. First, the initial weight of each bottle specimen was measured using an electronic chemical balance. For MVTR measurement, the bottle specimens were placed in a humidified chamber. The constant temperature and relative humidity were $40^{\circ} \mathrm{C}$ and $20 \%$, respectively. The weight of each specimen was measured as a function of time. Specifically, each bottle specimen was periodically removed from the chamber and weighed after 14 days. MVTR was calculated using the following formula:

$$
\begin{aligned}
& \text { MVRT(\%) } \\
& =\frac{\text { Initial weight }(\mathrm{g})-\text { Days later weight }(\mathrm{g})}{5 \mathrm{~g}} \times 100
\end{aligned}
$$

\subsection{Measurement of Residual L-Menthol}

The experimental setup for measuring the residual rate of L-menthol is illustrated in Figure 4. The nozzle specimens were placed in a glass ampoule and filled with 6 $\mathrm{mL}$ of $0.01 \mathrm{wt} \% \mathrm{~L}$-menthol. The glass ampoule was then heat sealed. Next, the ampoule was placed in a thermostatic oven at constant temperature of $50^{\circ} \mathrm{C}$. After 12 days, the ampoule was removed from the oven and the L-menthol content in the solution was measured via liquid chromatography (content after elapsed time). The residual rate of L-menthol was calculated using the following formula:

$$
\begin{aligned}
& \text { residual rate of L-menthol (\%) } \\
& =\frac{\text { Days later content }}{\text { Initial content }} \times 100
\end{aligned}
$$

\subsection{Mechanical Properties}

\subsubsection{Tensile Testing}

Dumbbell-shaped specimens were prepared from each molded sample. The tensile strength and elongation were

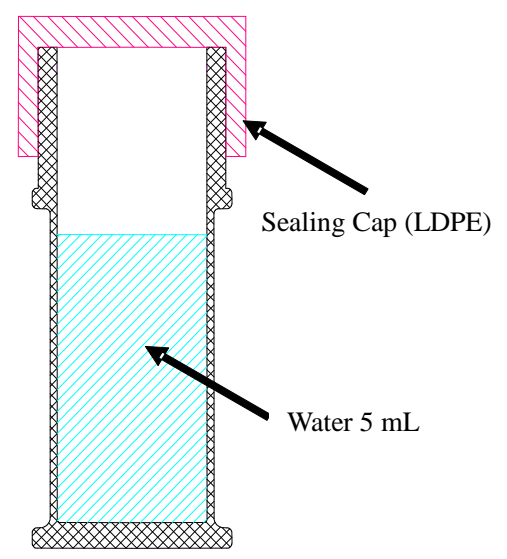

Figure 3. Scheme for MVTR measurement. measured using an Autograph Universal Testing Machine at $23^{\circ} \mathrm{C}$ and $100 \mathrm{~mm} / \mathrm{min}$.

\subsubsection{Compressive Testing}

Figure 5 shows a scheme of the experimental setup used for compressive testing. Compressive testing was conducted with a load of $150 \mathrm{~N}$ and a speed of $300 \mathrm{~mm} / \mathrm{min}$ on center of the cylindrical wall of each bottle sample.

\subsection{Morphology Studies}

Cross-sectional slices in the circumference direction of the cylindrical bottle specimens were examined using transmission electron microscopy (TEM).

\section{Results and Discussion}

\subsection{MVTR}

Figure 6 demonstrates the influence of PET content on the MVTR of the PET/PP blends after 14 days. It is clearly observed that MVTR significantly decreased with an increase in PP content. For pure PET, MVTR was $1.14 \%$; however, for $90 \%$ PET, MVTR reduced to $0.82 \%$

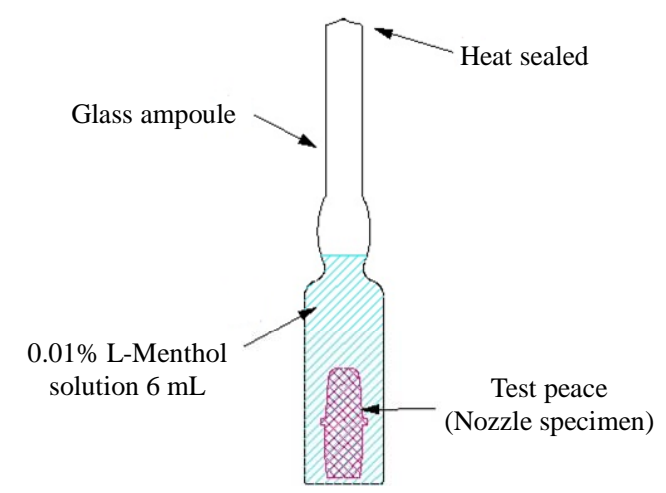

Figure 4. Scheme for measuring the residual rate of $L$ menthol.

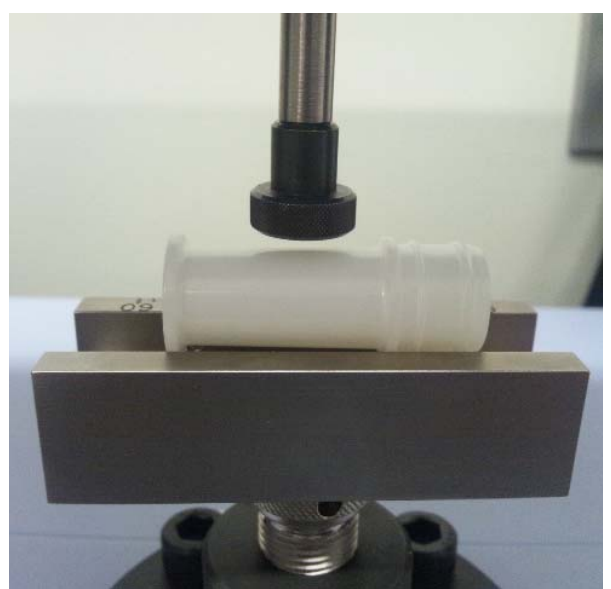

Figure 5. Scheme for compressive testing. 


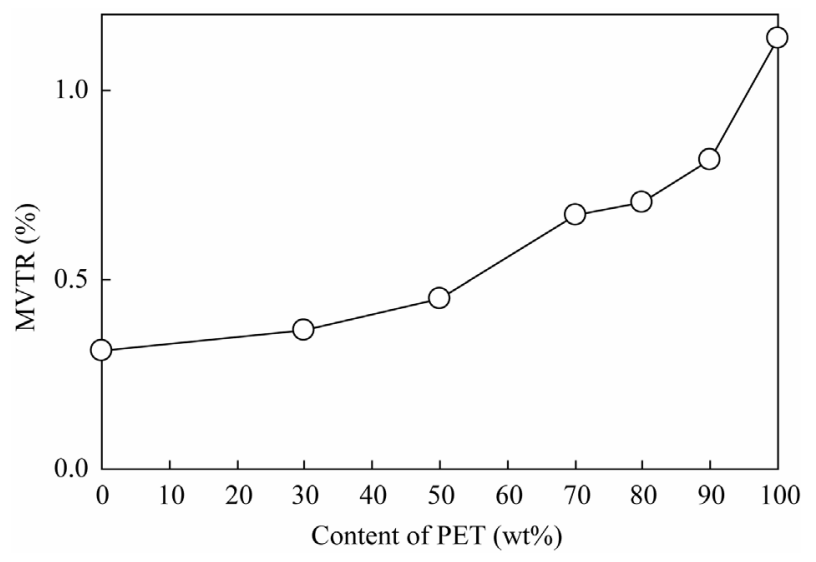

Figure 6. MVTR after 14 days.

at a dash. Thus, the MVTR of PET was improved by approximately $30 \%$ by blending with only $10 \mathrm{wt} \%$ of PP.

Similarly, between the $70 \%$ and $50 \%$ PET blends, the MVTR of PET was improved by approximately 33\% with blending of only $20 \mathrm{wt} \%$ of PP. The reduced rates observed between the 100\% PET and the 90\% PET blend and between the $70 \%$ and $50 \%$ PET blends demonstrated significant differences.

The MVTR results were considered in relation to specimen morphology (Figure 7). All blend materials exhibited a sea-island structure. For $90 \%, 80 \%$, and $70 \%$ PET blends, PP was dispersed with an elongated shape in the form of laminae and exhibited a lamellar structure. These results indicated that the barrier properties improved as a result of PP, which has a low MVTR, stratifying and dispersing within PET. Moreover, when the PP content was $50 \%$ or greater, the sea-island structure was reversed, and the barrier properties dramatically improved when PP became the sea layer. This indicates that the moisture barrier properties of the blends were effectively improved by the addition of a small amount of PP.

\subsection{Residual Rate of L-Menthol}

Figure 8 displays the influence of PET content in the PET/PP blends on the residual rate of L-menthol after 12 days. Residual rates of L-menthol in the $100 \%, 90 \%$, $80 \%$, and $70 \%$ PET specimens were unchanged. When the PET content was less than $50 \%$, the adsorption of L-menthol decreased with increasing PP content in the blend.

The residual rates of L-methanol were considered in relation to specimen morphology (Figure 7). PET was the sea layer in the $70 \%, 80 \%$, and $90 \%$ PET blends, indicating that the external layer exposed to L-menthol will be covered in PET. Hence, L-menthol was not adsorbed onto these blends. PP was the sea layer when the PET content was $50 \%$ and $30 \%$. Therefore, L-menthol

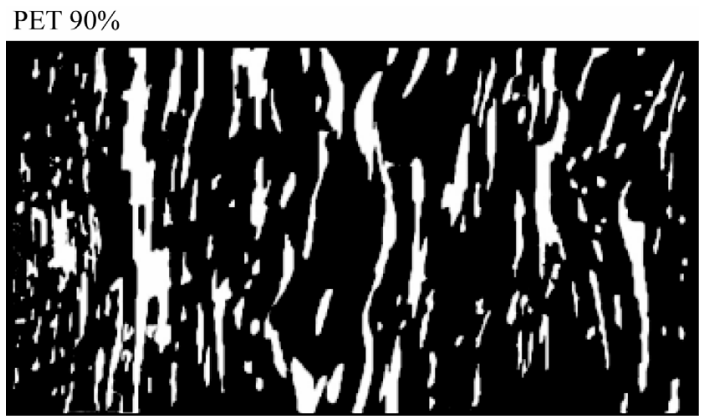

PET $80 \%$

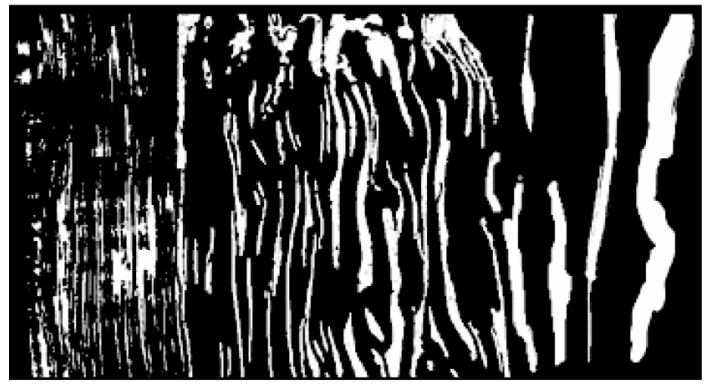

PET $70 \%$

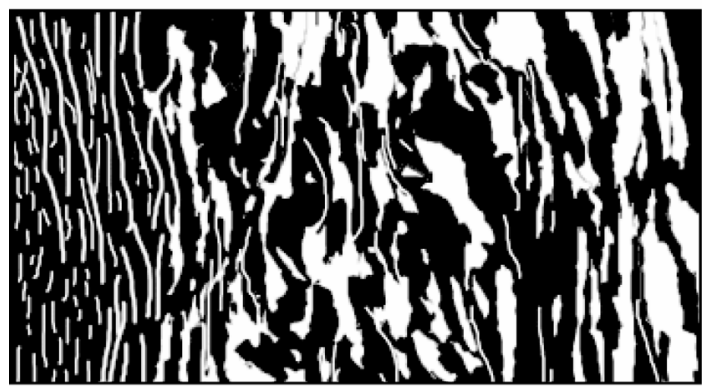

PET $50 \%$

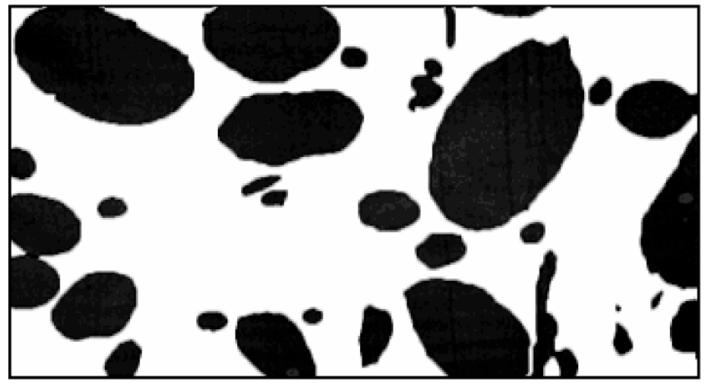

PET $30 \%$

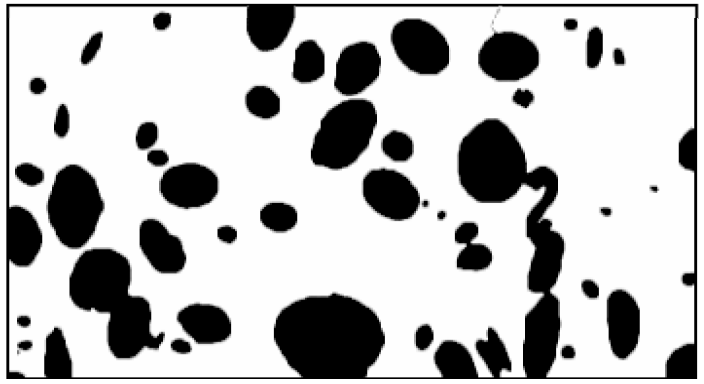

Figure 7. TEM images of PET/PP blends. 
adsorption was expected to occur. The anti-adsorptive property of $30 \%$ PET was almost similar to that of $100 \%$ PP. Thus, it is difficult to conclude whether the addition of $30 \%$ PET was effective for reducing adsorption.

\subsection{Mechanical Properties}

Figures 9 and $\mathbf{1 0}$ show the tensile strength and tensile elongation results for the PET/PP blends. PET has low elongation and high strength, whereas PP has high elongation and low strength. The tensile strength of the blended materials decreased with increasing PP content, while the tensile elongation of the blended materials decreased with increasing PET content. The results show that strength of the blended materials was significantly lowered due to poor compatibility between PET and PP.

Figure 11 shows the front views of the fractured area of specimens after compressive testing. Compressive testing was conducted to examine whether the blends were sufficiently strong to be used in eye-drop containers. Pure PET and pure PP did not break under compressive

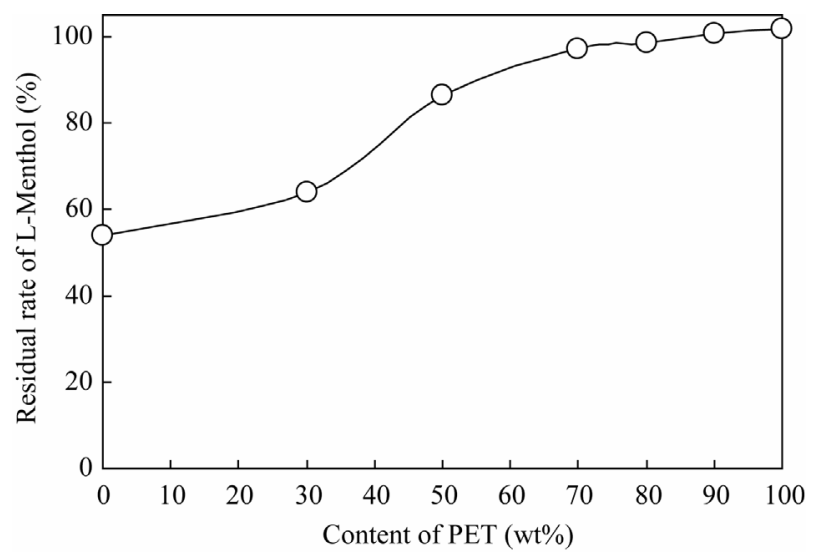

Figure 8. Residual rate of L-menthol after 12 days.

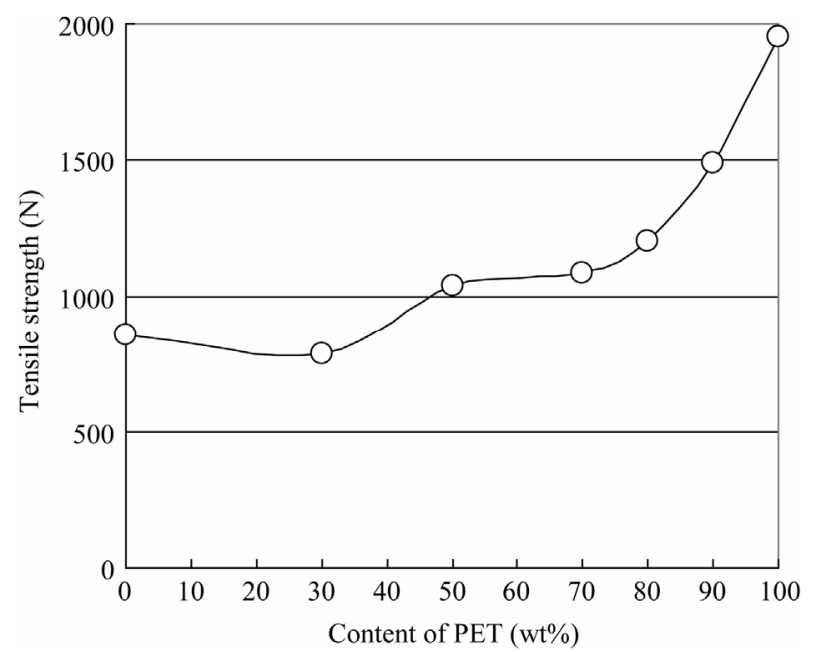

Figure 9. Tensile strength.

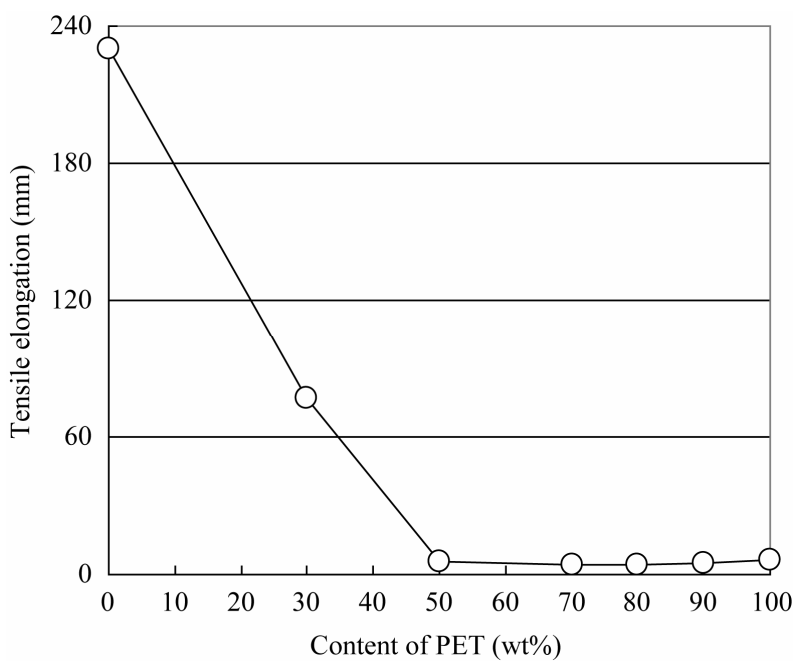

Figure 10. Tensile elongation.
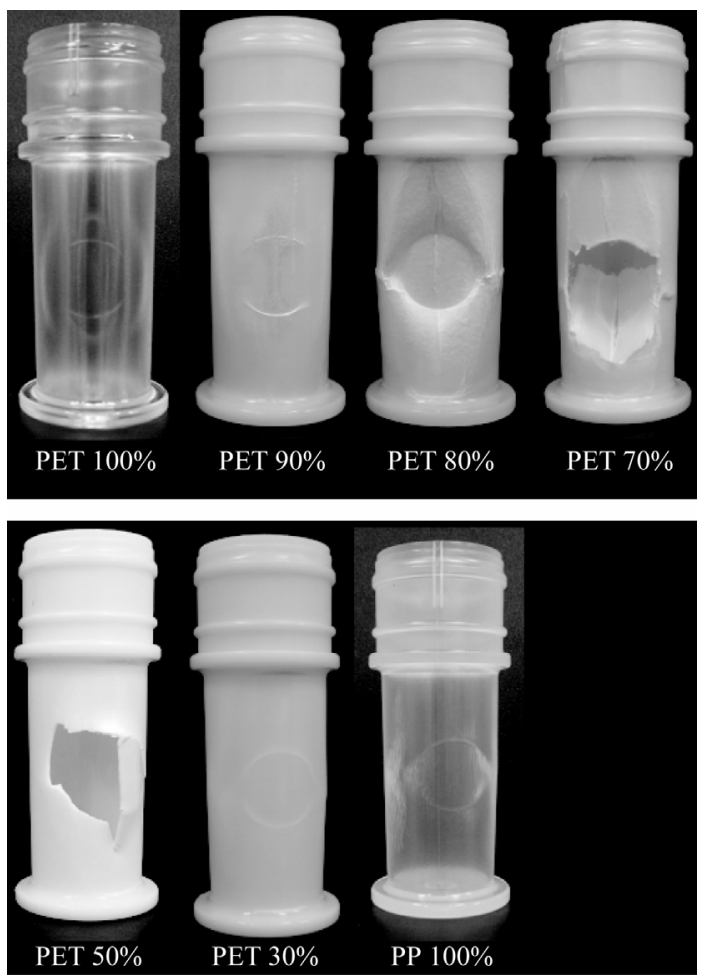

Figure 11. Representative images of samples after compressive testing.

testing. The $30 \%, 80 \%$, and $90 \%$ PET blends could bear the weight of compressive testing. However, the $50 \%$ and 70\% PET blends failed upon compression.

\section{Conclusions}

A balance in the anti-adsorptive and vapor barrier properties was achieved by blending PET and PP, with the exception of the PET/PP $=3 / 7$ blend. With respect to 
mechanical properties, the strength of PET/PP blends was reduced. However, the PET/PP blends, except the $\mathrm{PET} / \mathrm{PP}=7 / 3$ and PET $/ \mathrm{PP}=5 / 5$ blends, were able to bear the experimental load required for application in eye-drop containers.

Taken together, the results of this study indicate that the PET/PP = 9/1 and PET/PP = 8/2 blends are most applicable for use in eye-drop containers. In the future, methods are needed to improve the mechanical properties of the PET/PP $=5 / 5$ and PET/PP $=7 / 3$ blends, as they showed the most favorable balance between the MVTR and anti-adsorptive properties of L-menthol. As the next step, further experiments and analyses are required in terms of the solubility parameters of L-menthol, PET, and PP, which dominate the adsorption phenomena of the system.

\section{REFERENCES}

[1] X. L. Xu, N. N. Yu, Z. Z. Bai, Y. B. Xun, D. Jin, Z. J. Li and H. Cui, "Effect of Menthol on Ocular Drug Delivery," Graefe's Archive for Clinical and Experimental Ophthalmology, Vol. 249, No. 10, 2011, pp. 1503-1510. http://dx.doi.org/10.1007/s00417-011-1703-z

[2] M. G. Sajilata, K. Savitha, R. S. Singhal and V. R. Kanetkar, "Scalping of Flavors in Packaged Foods," Comprehensive Reviews in Food Science and Food Safety, Vol. 6, No. 1, 2007, pp. 17-35. http://dx.doi.org/10.1111/j.1541-4337.2007.00014.x

[3] C.-S. Deng, H. E. Assender, F. Dinelli, O. V. Kolosov, G. A. D. Briggs, T. Miyamoto and Y. Tsukahara, "Nucleation and Growth of Gas Barrier Aluminium Oxide on Surfaces of Poly(Ethylene Terephthalate) and Polypropylene: Effects of the Polymer Surface Properties," Journal of Polymer Science Part B: Polymer Physics, Vol. 38, No. 23, 2000, pp. 3151-3162. http://dx.doi.org/10.1002/1099-0488(20001201)38:23<31 51::AID-POLB150>3.0.CO;2-Q

[4] “Pharmaceutical Development Q8(R2),” International Conference on Harmonisation of Technical Requirements for Registration of Pharmaceuticals for Human Use, ICH
Harmonised Tripartite Guideline Current Step 4 Version, 2009.

http://www.ich.org/fileadmin/Public_Web_Site/ICH_Pro ducts/Guidelines/Quality/Q8_R1/Step4/Q8_R2_Guidelin e.pdf

[5] T. Kamako, M. Oya and T. Nishihata, "Prediction of Water Loss from Plastic Container for Eye-Drop in Various Storage Conditions," PDA Journal of GMP and Validation in Japan, Vol. 3, No. 2, 2001, pp. 97-103. http://dx.doi.org/10.11347/pda.3.97

[6] M. K. Cheung and D. Chan, "Mechanical and Rheological Properties of Poly(Ethylene Terephthalate)/Polypropylene Blends,” Polymer International, Vol. 43, No. 3, 1997, pp. 281-287.

http://dx.doi.org/10.1002/(SICI)1097-0126(199707)43:3< 281::AID-PI800>3.0.CO;2-Q

[7] M. Akbari, A. Zadhoush and M. Haghighat, "PET/PP Blending by Using PP-g-MA Synthesized by Solid Phase,” Journal of Applied Polymer Science, Vol. 104, No. 6, 2007, pp. 3986-3993. http://dx.doi.org/10.1002/app.26253

[8] C. Papadopoulou and N. Kalfoglou, "Comparison of Compatibilizer Effectiveness for PET/PP Blends: Their Mechanical, Thermal and Morphology Characterization,” Polymer, Vol. 41, No. 7, 2000, pp. 2543-2555. http://dx.doi.org/10.1016/S0032-3861(99)00442-5

[9] M. Pracella and D. Chionna, "Reactive Compatibilization of Blends of PET and PP Modified by GMA Grafting," Macromolecular Symposia, Vol. 198, No. 1, 2003, pp. 161-172. http://dx.doi.org/10.1002/masy.200350814

[10] M. Xanthos, M. W. Young and J. A. Biesenberger, "Polypropylene/Polyethylene Terephthalate Blends Compatibilized through Functionalization,” Polymer Engineering \& Science, Vol. 30, No. 6, 1990, pp. 355-365. http://dx.doi.org/10.1002/pen.760300607

[11] Y. X. Panga, D. M. Jiaa, H. J. Hua, D. J. Hourstonb and M. Songb, "Effects of a Compatibilizing Agent on the Morphology, Interface and Mechanical Behaviour of Polypropylene/Poly(Ethylene Terephthalate) Blends,” Polymer, Vol. 41, No. 1, 2000, pp. 357-365. http://dx.doi.org/10.1002/pen.760300607 